\title{
In-Hospital Cardiopulmonary Resuscitation of Patients With Human Immunodeficiency Virus Infection: A Population- Based Cohort Study of Epidemiology and Outcomes
}

\author{
Lavi Oud
}

\begin{abstract}
Background: Marked improvements were realized in both shortterm and long-term outcomes of human immunodeficiency virus (HIV)-infected patients following the introduction of combination antiretroviral therapy. However, the contemporary population-level patterns of in-hospital cardiopulmonary resuscitation (CPR) and the outcomes of HIV-infected patients were not systematically examined.

Methods: We used the Texas Inpatient Public Use Data File to identify hospitalizations aged $\geq 18$ years with and without HIV during 2009 - 2014, and those in each group who have undergone in-hospital CPR. Short-term survival (defined as absence of hospital mortality or discharge to hospice) following in-hospital CPR was examined. Multivariate logistic regression modeling was used to assess the prognostic impact of HIV infection following in-hospital CPR and predictors of short-term survival among HIV hospitalizations.
\end{abstract}

Results: In-hospital CPR was reported in 437 and 54,135 hospitalizations with and without HIV, respectively. The rates of in-hospital CPR (per 1,000 hospitalizations) were 4.4 and 4.1 among hospitalizations with and without HIV, respectively $(\mathrm{P}=0.1659)$. The corresponding rates of in-hospital CPR among decedents were $11 \%$ and $11.8 \%$, respectively $(\mathrm{P}=0.1531)$. Crude short-term survival following in-hospital CPR among hospitalizations with and without HIV was $19 \%$ and $26.8 \%$, respectively $(\mathrm{P}=0.0003)$. The corresponding adjusted short-term survival between 2009 and 2014 rose from $14.2 \%$ to $27 \%(\mathrm{P}=0.0009$ for trend $)$ and from $25.5 \%$ to $28 \%(\mathrm{P}<0.0001$ for trend). HIV infection was associated with lower odds of shortterm survival following in-hospital CPR (adjusted odds ratio (aOR): $0.50,95 \%$ confidence interval $(95 \% \mathrm{CI}): 0.39$ - 0.65$)$. Select comorbid conditions (congestive heart failure, aOR: 2.03, 95\% CI: 1.20 2.46; cerebrovascular disease, aOR: $2.08,95 \% \mathrm{CI}: 1.15$ - 3.75; and diabetes, aOR: $1.53,95 \%$ CI 1.31 - 4.71) were the only independent predictors of short-term survival following in-hospital CPR among HIV hospitalizations.

Manuscript submitted February 10, 2020, accepted March 9, 2020

Division of Pulmonary and Critical Care Medicine, Department of Internal Medicine, Texas Tech University Health Sciences Center at the Permian Basin, 701 W. 5th St., Odessa, TX 79763, USA.Email: lavi.oud@ttuhsc.edu

doi: https://doi.org/10.14740/jocmr4108
Conclusions: The rates of in-hospital CPR were similar among hospitalizations with and without HIV infection, with similar level of selectivity among decedents. Although HIV infection was associated with lower short-term survival following in-hospital CPR for the whole cohort, a dramatic improvement was observed during the study period among affected patients, with short-term survival rates becoming near-similar to those without HIV. Further studies are needed to identify modifiable factors to further improve the outcomes following in-hospital CPR among patients with HIV infection.

Keywords: Cardiopulmonary resuscitation; Human immunodeficiency virus; Hospitalization; Survival; Prognosis

\section{Introduction}

The long-term outlook for patients infected with human immunodeficiency virus (HIV) has been transformed over the past decades, following the introduction of combined antiretroviral therapy (cART) in the mid-1990s, from a consistently rapidly fatal disease to a chronic, manageable illness. As a result, people living with HIV can reach near-normal long-term survival in developed countries [1]. Concurrently, the rates of hospitalization [2,3] and hospital mortality [4] have progressively decreased over time among HIV-infected patients. In addition, the short-term prognosis among those requiring admission to the intensive care unit (ICU) became similar to that of critically ill patients in the general population $[5,6]$.

However, it is unclear whether the abovementioned gains in short-term outcomes in the HIV-infected population extend to those following cardiopulmonary resuscitation (CPR) for inhospital cardiac arrest. Hospital survival following in-hospital CPR has been progressively improving in the general population [7-9]. However, reports on the corresponding epidemiology and outcomes among hospitalized HIV-infected patients have been rare. Survival following CPR for in-hospital cardiac arrest has been remarkably poor in the early years following discovery of HIV, with $2.3 \%$ of patients surviving to hospital discharge in a single-center study and CPR in these patients has been considered potentially futile [10]. A recent multicenter study in France reported 25.5\% short-term mortality among HIV-infected patients following in-hospital CPR, and HIV infection was not associated with adverse impact on survival 
[11]. However, the investigators did not describe the rates of in-hospital CPR, and combined patients with out-of-hospital and in-hospital CPR in their reporting on patients' characteristics and modeling of survival [11]. Finally, the temporal trends of patients' survival were not examined.

An analysis of the patterns of CPR utilization following inhospital CPR among HIV-infected patients and the outcomes among the latter is important to better understand the current burden of this complication, for development of preventive and interventional programs to improve patient outcomes and, importantly, to inform clinicians' decision-making and discussions with patients and their surrogates about goals of care.

Here, we report a population-based study of hospitalized patients with and without HIV designed to: 1) examine the comparative patterns of utilization of in-hospital CPR and its associated outcomes, overall and over time; 2) characterize the patients undergoing in-hospital CPR; and 3) determine the prognostic impact of HIV infection following in-hospital CPR and the factors associated with short-term survival among those with HIV.

\section{Materials and Methods}

This was a retrospective, population-based cohort study. Because we used a publicly available, de-identified data set, the study was determined to be exempt from formal review by the Texas Tech Health Sciences Center's Institutional Review Board. A detailed description of the study methodology is included in the Supplementary Material 1 (www.jocmr.org). This cohort study adheres to the Strengthening the Reporting of Observational Studies in Epidemiology (STROBE) reporting guidelines [12].

\section{Data sources and study population}

We used the Texas Inpatient Public Use Data File (TIPUDF), an administrative data set maintained by the Texas Department of State Health Services [13]. The TIPUDF includes approximately $97 \%$ of hospital discharges in the state and its characteristics were previously described [14].

We initially extracted data on all patients aged $\geq 18$ years, who were hospitalized between the years 2009 and 2014. We then identified among these hospitalizations those with a diagnosis of HIV and further identified, among the latter, hospitalizations with in-hospital CPR. This latter group formed the primary analytic cohort. A diagnosis of HIV was based on the presence of International Classification of Diseases, Ninth Revision, Clinical Modification codes (ICD-9) 042, 079.53, 795.71, and V08 in the principal or secondary diagnosis fields. These codes were used in prior studies of HIV in administrative data [15-17]. CPR was identified based on the presence of ICD-9 codes 99.60 or $99.63[8,18]$. Hospitalizations with a principal diagnosis of cardiac arrest (ICD-9 code 427.5), ventricular fibrillation (ICD-9 code 427.41) or ventricular flutter (ICD-9 code 427.42) were excluded.

In order to identify secular trends not captured through analysis of the primary cohort, we anchored the data to similar- aged hospitalizations during 2009 - 2014 without a diagnosis of HIV who had in-hospital CPR. In addition, we have examined all hospitalizations aged $\geq 18$ years during the years 2009 - 2014 with and without a diagnosis of HIV and those who died during hospitalization within each group to estimate the annual rates of in-hospital CPR among all hospitalizations and among hospital decedents.

\section{Outcomes}

The co-primary outcomes were rates of in-hospital CPR among all hospitalizations with and without HIV and rates of short-term survival among hospitalizations with in-hospital CPR. We defined short-term survival as that of hospitalizations without in-hospital death or discharge to hospice.

The secondary outcomes included: 1) overall and over time rates of in-hospital CPR among all hospitalizations with and without HIV who died in the hospital; and 2) overall and over time short-term survival among those with and without shockable rhythm, and hospital disposition of those without in-hospital death or discharge to hospice, both among hospitalizations with and without HIV who have undergone in-hospital CPR.

\section{Study variables}

Study variables were selected based on clinical plausibility and prior reports $[4,8,18]$. The abstracted variables included: 1) demographics (age, gender, race/ethnicity, and health insurance); 2) comorbid conditions, based on the Deyo modification of the Charlson comorbidity index [19]; 3) admission during the weekend (vs. during weekdays); 4) presence of a shockable rhythm (ICD-9 codes 427.41 or 427.42) [8, 20] (these codes were reported to have positive predictive values between $74 \%$ [21] and 100\% [22] in administrative data); 5) number of failing organs [23]; 6) sepsis; 7) hospitals' teaching status; 8) hospital disposition; and 9) year of admission.

\section{Data analysis}

Categorical variables were summarized as frequencies and percentages, and continuous variables were reported as means (standard deviation (SD)). Group comparisons were performed using Chi-square test for categorical variables and $t$ test or Mann-Whitney test for continuous variables. We used hospitalizations as the unit of analysis because the TIPUDF does not identify individual patients and thus does not capture repeated hospitalizations.

In order to provide further anchoring context to the trends of outcome trajectories between hospitalizations with and without HIV who had in-hospital CPR, we examined their respective temporal trends of the burden of chronic illness and illness severity using the Deyo comorbidity index and the number of failing organs, respectively, as proxy measures.

Temporal trends of the primary and secondary outcomes were examined using weighted least square regression, with 
calendar year as predictor. Modeled findings are reported as average annual percent change (AAPC) and 95\% confidence intervals $(95 \% \mathrm{CI})$. All temporal analyses were carried out separately for hospitalizations with and without HIV. Comparison of regression slopes was performed using the methods described by Armitage et al [24].

We estimated the risk-adjusted of short-term survival following in-hospital CPR among hospitalizations with and without HIV using empirical Bayesian posterior estimates from multivariate logistic regression models for each (see modeling approach below) and then derived the corresponding annual rates of short-term survival.

A multivariate logistic regression model was fitted to estimate the association between HIV as independent predictor and short-term survival following in-hospital CPR as dependent variable. Predictors with $\mathrm{P}<0.1$ on univariate logistic regression were considered for multivariate analysis, following examination for multicollinearity. Candidate predictor covariates were then entered into the model using backward stepwise selection. Results of the multivariate models were expressed as adjusted odds ratio (aOR) and their $95 \% \mathrm{CI}$.

The potential predictors of short-term survival following in-hospital CPR among hospitalizations with HIV were examined using multivariate logistic regression modeling, employing the approach described above for the whole cohort.

Data analyses were carried out on MedCalc version 17.5.5 (MedCalc Software, Ostend, Belgium). A two-sided P value < 0.05 was considered statistically significant.

\section{Results}

The total number of hospitalizations with and without HIV during the study period was 99,657 and $13,192,847$, respectively. There were 3,125 in-hospital deaths among hospitalizations with HIV and 286,173 deaths among those without HIV, corresponding hospital mortality rates of $3.1 \%$ and $2.2 \%$, respectively. The details of the annual hospitalization volumes and the corresponding hospital mortality of all hospitalizations with and without HIV are provided (Supplementary Material 2, www.jocmr.org). Hospital mortality did not change significantly over time in either group (AAPC $(95 \% \mathrm{CI})$ : $+4.4(-9.9$ to +1.1$) ; \mathrm{P}=0.0922$ for hospitalizations with HIV and $0(-1.8$ to +1.8$) ; \mathrm{P}=1.000$ for hospitalizations without HIV).

\section{Utilization of in-hospital CPR, patient characteristics, and short-term survival}

There were 54,572 hospitalizations with in-hospital CPR during the study period, of which 437 were with a diagnosis of HIV and 54,135 without HIV. The characteristics of these hospitalizations are detailed in Table 1. In-hospital CPR was reported among hospitalizations with and without HIV in 4.4 and 4.1 per 1,000 hospitalizations, respectively $(\mathrm{P}=0.1659)$ and in $11.0 \%$ vs. $11.8 \%$, respectively, among hospital decedents $(\mathrm{P}=0.1531)$.

The short-term survival following in-hospital CPR was markedly lower among hospitalizations with HIV than among those without HIV (19.0\% vs. $26.8 \%$, respectively; $\mathrm{P}=0.0003$ ). When stratified by the presence or absence of a shockable rhythm, short-term survival was similar between the two groups among hospitalizations with HIV (19.4\% vs. $19.0 \%$, respectively; $\mathrm{P}=0.9576$ ), while being markedly higher among those with shockable rhythm among hospitalizations without HIV ( $36.2 \%$ vs. $25.5 \%$, respectively; $\mathrm{P}<0.0001)$.

\section{Temporal trends among hospitalizations with in-hospital CPR}

The temporal patterns of the rates of in-hospital CPR, presence of a shockable rhythm, and related short-term survival among hospitalizations with and without HIV are described in Table 2 . The details of the annual volumes of corresponding strata are provided (Supplementary Material 2, www.jocmr.org). The temporal trends of risk-adjusted short-term survival following in-hospital CPR among hospitalizations with and without HIV are presented in Figure 1.

The annual rates of in-hospital CPR remained unchanged among hospitalizations with HIV, while rising on average by $2.7 \% /$ year among those without HIV. Similarly, the annual rates of in-hospital CPR among hospital decedents remained unchanged among hospitalizations with HIV, while rising slightly, but statistically significantly, among those without HIV. However, there has been no significant difference in the slopes of the rates of in-hospital CPR over time between those with and without HIV infection for the whole cohort or among decedents ( $\mathrm{P}$ values for slope comparisons: $\mathrm{P}=0.1181$ and $\mathrm{P}=0.3312$, respectively).

The crude short-term survival following in-hospital CPR rose among hospitalizations with or without HIV, but did not reach statistical significance among the latter (Table 2). Similarly, the risk-adjusted short-term survival following in-hospital CPR improved among hospitalizations with and without HIV. However, the pace of improvement was greater in the HIV group vs. those without HIV (Fig. 1), increasing between 2009 and 2014 from $14.2 \%$ to $27.0 \%$, respectively, as compared with $25.5 \%$ vs. $28.0 \%$, respectively. The Deyo comorbidity index and the number of organ failures rose progressively over the study period among hospitalizations with and without HIV who have undergone in-hospital CPR, though the rise of the Deyo comorbidity index was not statistically significant among those with HIV. However, there was no difference between the slopes of either measure between hospitalizations with and without HIV (Supplementary Material 3, www.jocmr.org).

The annual rates of discharge to hospice following in-hospital CPR remained unchanged among hospitalizations with HIV (AAPC $=+57.5$, (95\% CI: -15.9 to +130.1$) ; \mathrm{P}=0.0952)$, while rising 5.9\%/year among those without HIV (95\% CI: 2.8 to $9.0 ; \mathrm{P}=0.0064$ ).

\section{Predictors of short-term survival}

The predictors of short-term survival following in-hospital CPR for the whole cohort and among hospitalizations with 
Table 1. The Characteristics of Hospitalizations With In-Hospital Cardiopulmonary Resuscitation, With and Without HIV

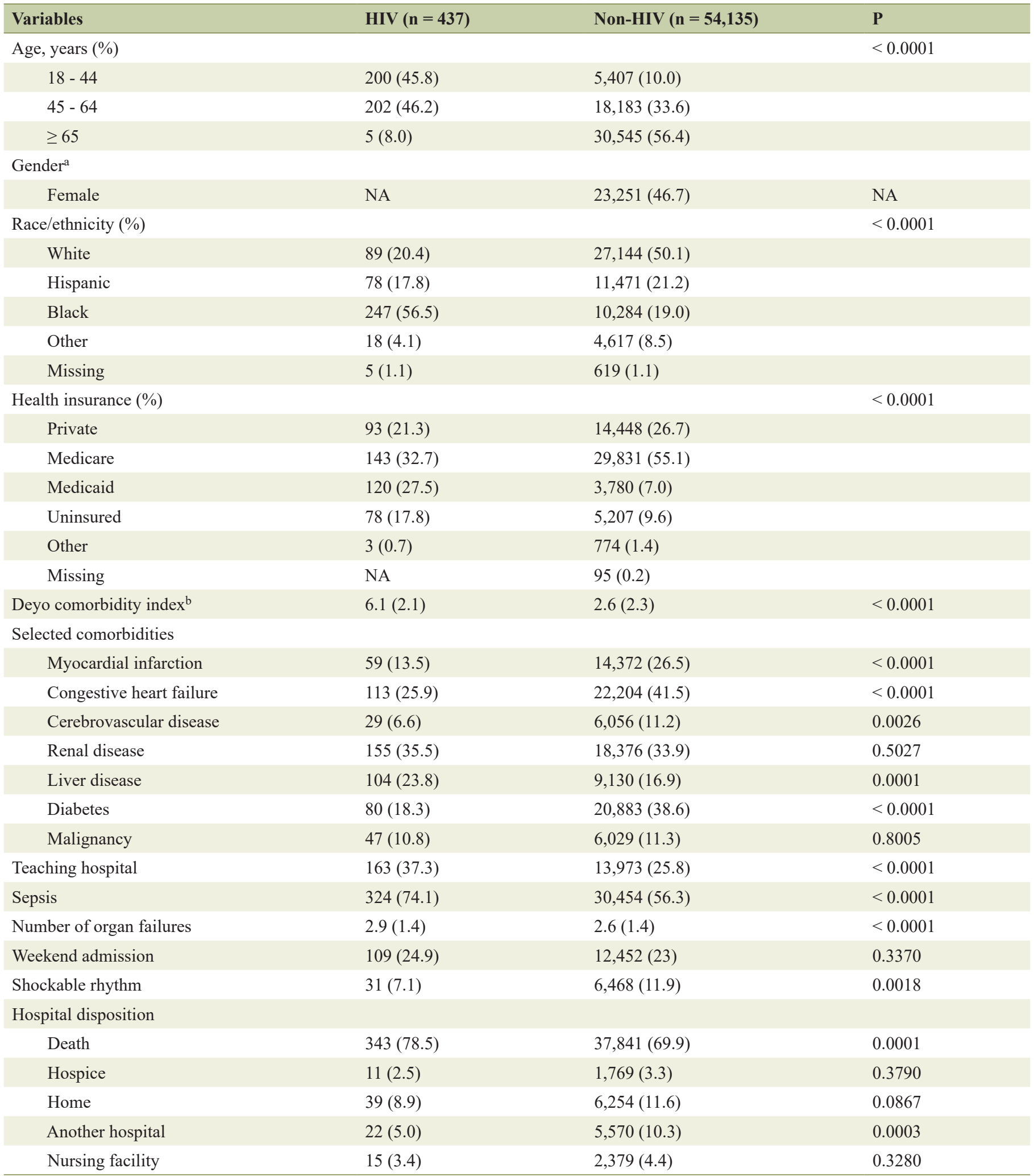

HIV: human immunodeficiency virus; SD: standard deviation. aGender is not reported for HIV hospitalizations and was reported in 49,777 hospitalizations without HIV; the percent figure for gender among the latter refers to that column's denominator for gender. ${ }^{\mathrm{b}} \mathrm{Mean}$ (SD). Percentage figures may not add to 100 due to rounding. 
Table 2. Temporal Changes Among Hospitalizations With In-Hospital Cardiopulmonary Resuscitation, With and Without HIV

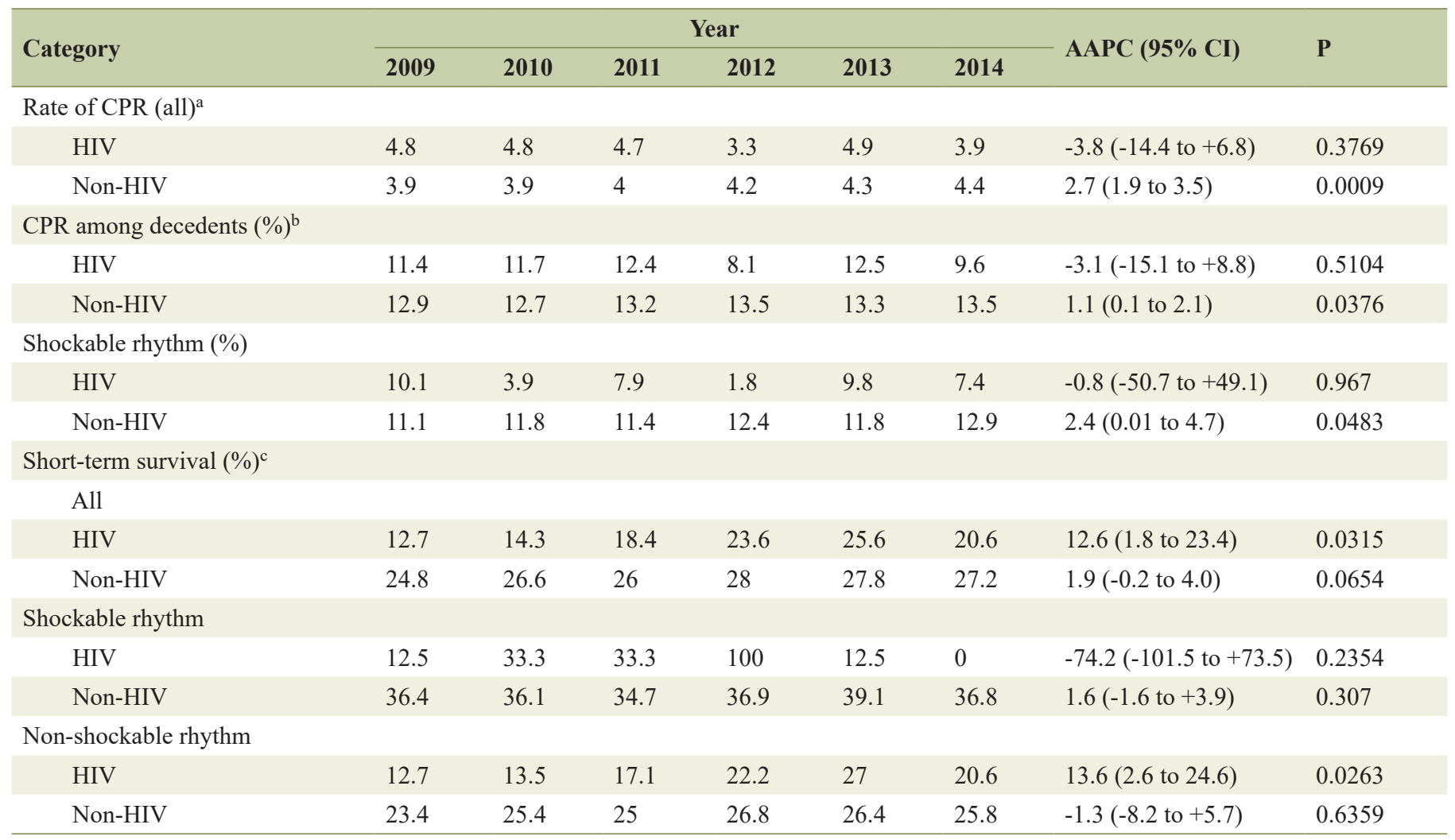

AAPC: average annual percent change; $95 \% \mathrm{Cl}$ : 95\% confidence interval; HIV: human immunodeficiency virus. ${ }^{\text {aRate }}$ of cardiopulmonary resuscitation per 1,000 hospitalizations among all hospitalizations within a specific stratum. ${ }^{\text {bPercent }}$ of cardiopulmonary resuscitation among all patients who died in the hospital within a specific stratum. 'Short-term survival was defined as that of hospitalizations without in-hospital death or discharge to hospice.

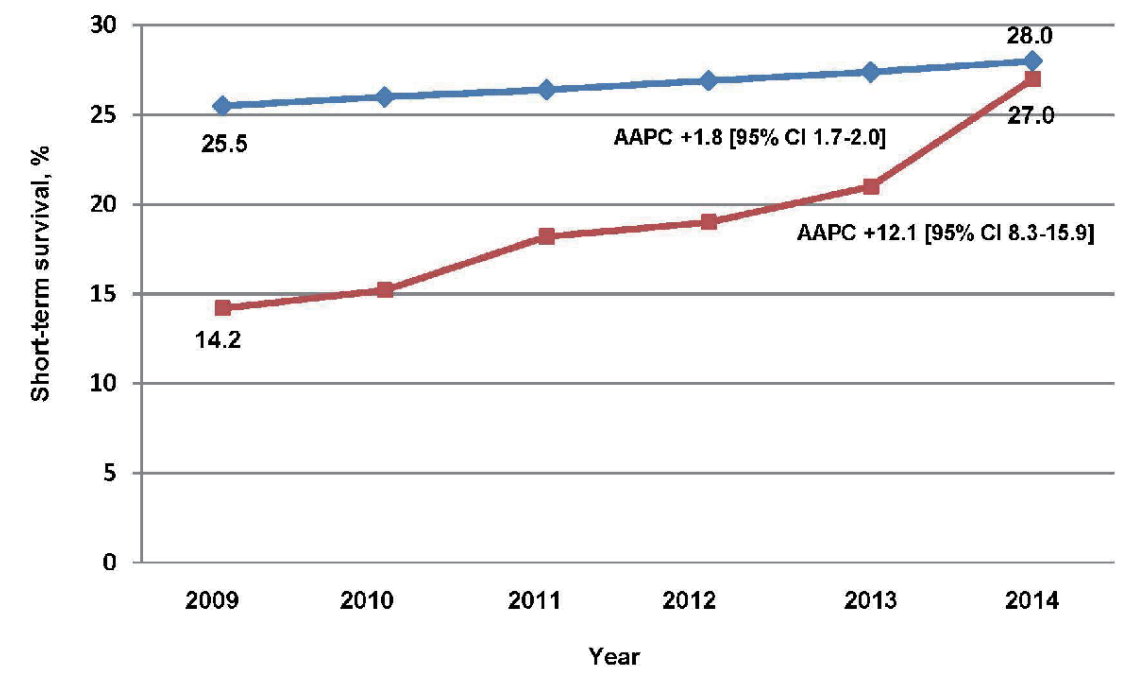

Figure 1. Temporal trends of risk-adjusted short-term survival following in-hospital cardiopulmonary resuscitation. Short-term survival is defined as that of hospitalizations without in-hospital death or discharge to hospice. Hospitalizations with HIV are represented by box markers, and those without HIV are represented by diamond markers. Short-term survival estimates were adjusted for age, race/ethnicity, health insurance, Deyo comorbidity index, myocardial infarction, cerebrovascular disease, diabetes, weekend admission, sepsis, shockable rhythm, number of organ failures, and year of admission. HIV: human immunodeficiency virus; $95 \% \mathrm{Cl}$ : 95\% confidence interval; AAPC: average annual percent change. 
Table 3. Univariate and Multivariate Logistic Regression Analysis of Predictors of Short-Term Survival Among All Hospitalizations With In-Hospital Cardiopulmonary Resuscitation

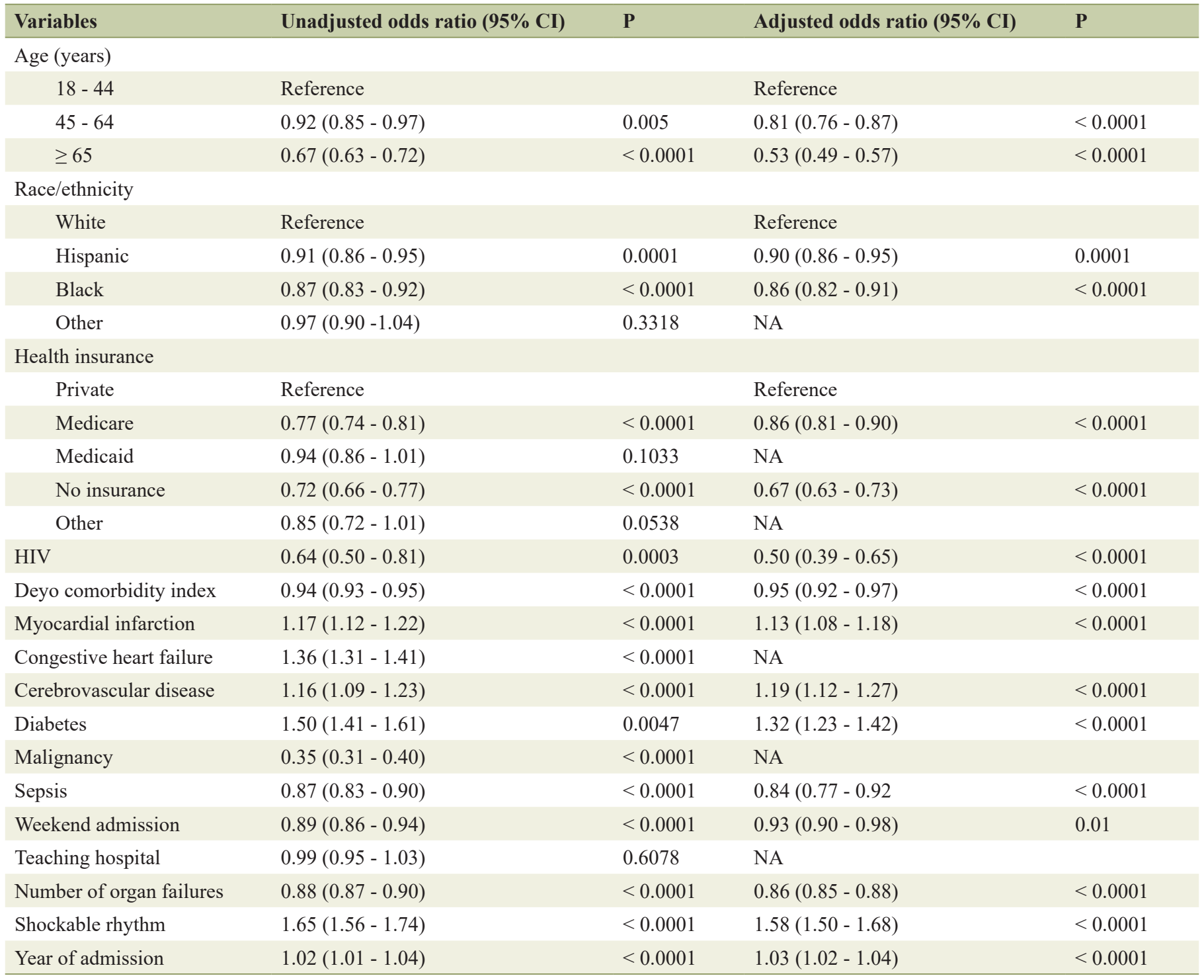

HIV: human immunodeficiency virus; 95\% Cl: 95\% confidence interval.

HIV are described in Tables 3 and 4, respectively.

HIV infection was associated with 50\% lower odds of short-term survival following in-hospital CPR on adjusted analysis. Among hospitalizations with HIV who had in-hospital CPR, only congestive heart failure, cerebrovascular disease, diabetes, and the year of admission were independent predictors, and were associated with higher odds of short-term survival. The predictors of short-term survival following inhospital CPR among hospitalizations without HIV are detailed (Supplementary Material 4, www.jocmr.org).

\section{Discussion}

The patterns of utilization of in-hospital CPR among HIV- infected patients have not been systematically examined, and the outcomes of affected patients have not been widely reported. In this population-based study, in-hospital CPR was used in 4.4 per 1,000 HIV-infected hospitalizations and in one in nine HIV-infected decedents, with both rates being similar to their counterparts without HIV. Short-term survival following in-hospital CPR was markedly lower among HIVinfected hospitalizations and HIV infection was associated with lower survival. However, short-term survival following in-hospital CPR rose rapidly among HIV-infected hospitalizations, reaching near-similar rates on adjusted analyses by the end of the study period. No modifiable factors were identified on examination of predictors of short-term survival following in-hospital CPR among HIV-infected hospitalizations. 
Table 4. Univariate and Multivariate Logistic Regression Analysis of Predictors of Short-Term Survival Following In-Hospital Cardiopulmonary Resuscitation Among Hospitalizations With HIV

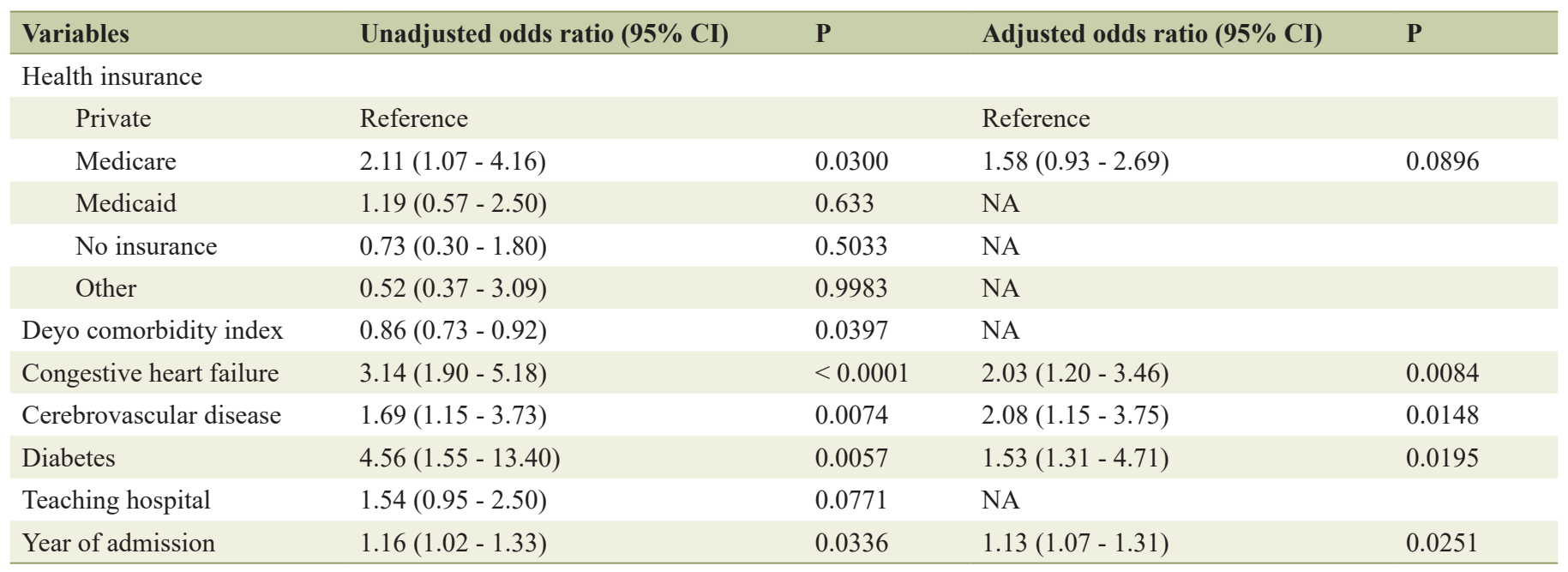

HIV: human immunodeficiency virus; $95 \% \mathrm{Cl}$ : $95 \%$ confidence interval.

\section{Relationship to prior studies}

This study provides, to our knowledge, the first population-level examination the cross-sectional and longitudinal patterns of CPR utilization for in-hospital cardiac arrest among HIV-infected patients. Our finding of similar rates of in-hospital CPR among hospitalizations with and without HIV may suggest that both patients (and their surrogates) and physicians do not consider inhospital CPR a futile intervention among the former, given the overall marked improvement in the longevity of patients with HIV. The similar rates of in-hospital CPR among decedents with and without HIV infection indicate comparative level of selectivity in using in-hospital CPR and further support this postulate.

Short-term survival following in-hospital CPR was over eight times higher in the present study as compared to that reported in the early years following identification of HIV (19\% vs. $2.3 \%$ [10]), though still markedly lower than that among contemporary non-HIV hospitalizations. The factors underlying the worse outcome among the former are unclear. Some of the potential contributors to the lower short-term survival among those with HIV infection include higher burden of chronic illness, higher severity of illness, and higher rates of sepsis; and possibly differences in the proximal causes of resuscitated in-hospital cardiac arrest may have differed between those with and without HIV. However, while plausible, the abovementioned potential factors do not adequately explain the overall survival gaps, given the temporal trends in shortterm survival (see further discussion below).

Similarly, the markedly lower frequency of a shockable rhythm among the HIV-infected hospitalizations does not explain their survival gap since short-term survival was similar regardless of presence or absence of a shockable rhythm, in contrast with the well-documented prognostic advantage of a shockable rhythm in the general population $[8,9]$.

HIV infection was independently associated with $50 \%$ lower odds of short-term survival following in-hospital CPR, as compared to those without HIV, contrasting with finding of lack of association of HIV infection with post-CPR survival in a recent study by Mongardon and colleagues [11]. However, the latter study, analyzing the outcomes of only 28 HIV-infected patients, combined the outcomes of patients with out-ofhospital and in-hospital cardiac arrest; and the patient mix and processes of care may have differed between the two studies.

Despite the overall lower short-term survival following inhospital CPR among HIV hospitalizations, a key finding of the present study is the dramatic, $12 \%$ /year, rise in risk-adjusted short-term survival in this group, becoming by the end of the study period near-similar to that of hospitalizations without HIV (27\% vs. 28\%, respectively). This rapid improvement, compared to the much slower pace $(1.8 \%$ /year) among hospitalizations without HIV, took place despite similar rise over time in illness severity in both groups. The sources of the rapid improvement in the short-term survival among HIV-infected hospitalizations in the preset study cannot be directly determined from the administrative data. Improvements in postCPR care in general would be expected to be slower than those observed in the present study. Alternatively, it is possible that the rising use of cART [25] has contributed to the improved short-term survival among HIV-infected hospitalizations that had undergone in-hospital CPR. The latter factor would be expected to improve hospital mortality among all HIV hospitalizations and those with in-hospital CPR. However, hospital mortality remained unchanged over time among all HIV hospitalizations during the study period, thus casting doubt on the magnitude of the contribution of increased cART use.

The short-term survival following in-hospital CPR among HIV-infected hospitalizations in the present study was markedly lower than that reported by Mongardon et al (19\% vs. $25.5 \%$ [11], respectively). However, there are several major differences in the design and reporting between the two studies that likely contributed to the reported survival gap. First, the cohort in the latter study was restricted to ICU-treated patients. Thus, patients who were not admitted to ICU following in- 
hospital CPR or did not have a return of spontaneous circulation were not included. Second, the investigators restricted their outcome reporting to ICU mortality. Together, these factors have likely resulted in overestimation of survival, as compared to the all-inclusive design of the present study and use of hospital death or discharge to hospice as the primary survival outcome. Finally, as noted earlier, the patient characteristics and process of care may have differed between the two studies.

A striking finding of the present study was the markedly limited number of factors that were independently associated with short-term survival following in-hospital CPR among hospitalizations with HIV, in contrast to their non-HIV counterparts. Specifically, commonly impactful sociodemographic factors (e.g., age, race/ethnic, health insurance), the number of failing organs, and sepsis no longer affected HIV-infected patients' short-term survival following CPR for in-hospital cardiac arrest. The factors underlying these latter observations, likely substantially driven by peri-arrest pathobiology of HIVinfected patients warrant further study.

\section{Implications of study findings}

The dramatic improvement of short-term survival following in-hospital CPR among HIV-infected patients in the present study suggests that it is reaching parity with post-CPR outcomes in the general population, in line with the gains made in all HIV hospitalizations and those with critical illness. The comparable level selectivity in utilization of in-hospital CPR among decedents with and without HIV indicates that clinicians and, by extension, HIV-infected patients and their surrogates generally view the potential benefits of in-hospital CPR similarly to those without HIV infection.

Identifying potentially modifiable prognostic factors may further improve the outcomes of HIV-infected patients undergoing in-hospital CPR. However, the lack of such identifiable factors in the present study, and specifically, lack of prognostic impact of factors such as race/ethnicity, the type of health insurance, or sepsis, poses a challenge to implementation of workable preventive and interventional efforts in this population. Further studies, using more granular data, are needed to better understand the unique factors precipitating in-hospital cardiac arrest among HIV-infected patients who undergo CPR, as well as the corresponding CPR- and post-CPR processes of care.

Our finding of temporally divergent trajectories of discharge to hospice among hospitalizations with and without HIV who had in-hospital CPR underscores the need for caution when interpreting the longitudinal patterns of hospital mortality-based outcomes in the general population and in comparative population studies, given the dynamic changes in illness complexity and discharge practices.

\section{Study strengths and limitations}

The present study is the largest to date on in-hospital CPR among HIV-infected patients. The use of a statewide, all-payer, high-quality data set of consecutive hospitalizations allowed transcending of local variations in case mix and practice patterns. In addition, the larger number of hospitalizations permitted more comprehensive examination of the spatial and temporal aspects of the epidemiology and outcomes of HIV-infected patients following in-hospital CPR.

Nevertheless, the study has several important limitations, in addition to those noted earlier, related mostly to its retrospective design and use of administrative data. First, use of administrative data may have led to misclassification of some of the examined hospitalizations. However, similar approach was used in prior other epidemiological studies $[8,15,16]$. Second, although we adjusted for a broad array of patient- and hospital-level covariates, the dataset did not include information on the duration of HIV infection, use of cART, control of HIV replication, $\mathrm{CD} 4^{+}$ T-cell counts, the hospital setting of cardiac arrest (e.g., general wards vs. monitored beds, or ICU) or, as noted earlier, the processes of care during and following CPR. Thus, residual confounding cannot be excluded. Last, it is unknown whether our observations reflect those in other states or nationally.

\section{Conclusions}

In-hospital CPR was utilized at similar rates among hospitalizations with and without HIV infection. Although HIV infection was associated with worse outcomes, a dramatic improvement of short-term survival following in-hospital CPR was observed among hospitalizations with HIV, nearly matching the survival of those without HIV by the end of the study period. Additional studies are warranted to identify modifiable factors to inform future efforts to improve the outcomes of $\mathrm{HIV}$-infected patients undergoing in-hospital CPR.

\section{Supplementary Material}

Suppl 1. Detailed Description of the Study Methodology.

Suppl 2. The Annual Volume of Hospitalizations With and Without HIV, Overall and for Specific Strata.

Suppl 3. Linear Regression of the Annual Changes in the Deyo Comorbidity Index and the Number of Organ Failures Among Hospitalizations With and Without HIV, Who Have Undergone In-Hospital Cardiopulmonary Resuscitation.

Suppl 4. Univariate and Multivariate Logistic Regression Analysis of Predictors of Short-Term Survival Following InHospital Cardiopulmonary Resuscitation Among Hospitalizations Without HIV.

\section{Acknowledgments}

None to declare.

\section{Financial Disclosure}

None to declare. 


\section{Conflict of Interest}

None to declare.

\section{Informed Consent}

Not applicable.

\section{Author Contributions}

Lavi Oud: design of work, acquisition of data, analysis and interpretation, composition, drafting, revising, editing and final approval.

\section{Data Availability}

The data supporting the findings of this study have been deposited in the Texas Inpatient Public Use Data File, Texas Department of State Health Services, Center for Health Statistics, Austin, Texas, and can be accessed from http://www.dshs. state.tx.us/ thcic/hospitals/Inpatientpudf.shtm.

\section{References}

1. Lerner AM, Eisinger RW, Fauci AS. Comorbidities in persons with HIV: the lingering challenge. JAMA. 2020;323(1):19-20.

2. Heslin $\mathrm{KC}$, Elixhauser A. HIV hospital stays in the United States, 2006-2013. HCUP Statistical Brief \#206. June 2016. Agency for healthcare research and quality. Available from: http://www.hcup-us.ahrq.gov/reports/statbriefs/ sb206-HIV-Hospital-Stays-Trends.pdf.

3. Navon L. Hospitalization Trends and Comorbidities Among People With HIV/AIDS Compared With the Overall Hospitalized Population, Illinois, 2008-2014. Public Health Rep. 2018;133(4):442-451.

4. Cowell A, Shenoi SV, Kyriakides TC, Friedland G, Barakat LA. Trends in hospital deaths among human immunodeficiency virus-infected patients during the antiretroviral therapy era, 1995 to 2011. J Hosp Med. 2015;10(9):608614.

5. Dickson SJ, Batson S, Copas AJ, Edwards SG, Singer M, Miller RF. Survival of HIV-infected patients in the intensive care unit in the era of highly active antiretroviral therapy. Thorax. 2007;62(11):964-968.

6. Mendez-Tellez PA, Damluji A, Ammerman D, Colantuoni E, Fan E, Sevransky JE, Shanholtz C, et al. Human immunodeficiency virus infection and hospital mortality in acute lung injury patients. Crit Care Med. 2010;38(7):15301535 .

7. Kazaure HS, Roman SA, Sosa JA. Epidemiology and outcomes of in-hospital cardiopulmonary resuscitation in the United States, 2000-2009. Resuscitation. 2013;84(9):12551260 .
8. Mallikethi-Reddy S, Briasoulis A, Akintoye E, Jagadeesh $\mathrm{K}$, Brook RD, Rubenfire M, Afonso L, et al. Incidence and survival after in-hospital cardiopulmonary resuscitation in nonelderly adults: US experience, 2007 to 2012. Circ Cardiovasc Qual Outcomes. 2017;10(2):e003194.

9. Girotra S, Nallamothu BK, Spertus JA, Li Y, Krumholz HM, Chan PS, American Heart Association Get with the Guidelines-Resuscitation I. Trends in survival after in-hospital cardiac arrest. N Engl J Med. 2012;367(20):19121920.

10. Raviglione MC, Battan R, Taranta A. Cardiopulmonary resuscitation in patients with the acquired immunodeficiency syndrome. A prospective study. Arch Intern Med. 1988;148(12):2602-2605.

11. Mongardon N, Geri G, Deye N, Sonneville R, Boissier F, Perbet S, Camous L, et al. Etiologies, clinical features and outcome of cardiac arrest in HIV-infected patients. Int J Cardiol. 2015;201:302-307.

12. von Elm E, Altman DG, Egger M, Pocock SJ, Gotzsche PC, Vandenbroucke JP, Initiative S. The Strengthening the Reporting of Observational Studies in Epidemiology (STROBE) statement: guidelines for reporting observational studies. PLoS Med. 2007;4(10):e296.

13. Texas inpatient public use data file. Texas Department of State Health Services, Center for Health Statistics, Austin, Texas. Available from: http://www.dshs.state.tx.us/ thcic/hospitals/Inpatientpudf.shtm.

14. Oud L, Watkins P. Contemporary trends of the epidemiology, clinical characteristics, and resource utilization of necrotizing fasciitis in Texas: a population-based cohort study. Crit Care Res Pract. 2015;2015:618067.

15. Lazar R, Kersanske L, Xia Q, Daskalakis D, Braunstein SL. Hospitalization rates among people with HIV/AIDS in New York City, 2013. Clin Infect Dis. 2017;65(3):469476.

16. Berry SA, Fleishman JA, Moore RD, Gebo KA, Network HIVR. Trends in reasons for hospitalization in a multisite United States cohort of persons living with HIV, 20012008. J Acquir Immune Defic Syndr. 2012;59(4):368375.

17. Crowell TA, Gebo KA, Blankson JN, Korthuis PT, Yehia BR, Rutstein RM, Moore RD, et al. Hospitalization rates and reasons among HIV elite controllers and persons with medically controlled HIV infection. J Infect Dis. 2015;211(11):1692-1702.

18. Stapleton RD, Ehlenbach WJ, Deyo RA, Curtis JR. Longterm outcomes after in-hospital CPR in older adults with chronic illness. Chest. 2014;146(5):1214-1225.

19. Quan H, Li B, Couris CM, Fushimi K, Graham P, Hider $\mathrm{P}$, Januel JM, et al. Updating and validating the Charlson comorbidity index and score for risk adjustment in hospital discharge abstracts using data from 6 countries. Am J Epidemiol. 2011;173(6):676-682.

20. Shahreyar M, Dang G, Waqas Bashir M, Kumar G, Hussain J, Ahmad S, Pandey B, et al. Outcomes of in-hospital cardiopulmonary resuscitation in morbidly obese patients. JACC Clin Electrophysiol. 2017;3(2):174-183.

21. Hennessy S, Leonard CE, Freeman CP, Deo R, Newcomb C, Kimmel SE, Strom BL, et al. Validation of diagnostic 
codes for outpatient-originating sudden cardiac death and ventricular arrhythmia in Medicaid and Medicare claims data. Pharmacoepidemiol Drug Saf. 2010;19(6):555-562.

22. Chung CP, Murray KT, Stein CM, Hall K, Ray WA. A computer case definition for sudden cardiac death. Pharmacoepidemiol Drug Saf. 2010;19(6):563-572.

23. Martin GS, Mannino DM, Eaton S, Moss M. The epidemiology of sepsis in the United States from 1979 through
2000. N Engl J Med. 2003;348(16):1546-1554.

24. Armitage P, Berry G, Matthews JNS. Statistical methods in medical research. 4th ed. Blackwell Science, 2002.

25. Forsythe SS, McGreevey W, Whiteside A, Shah M, Cohen J, Hecht R, Bollinger LA, et al. Twenty years of antiretroviral therapy for people living with HIV: global costs, health achievements, economic benefits. Health Aff (Millwood). 2019;38(7):1163-1172. 